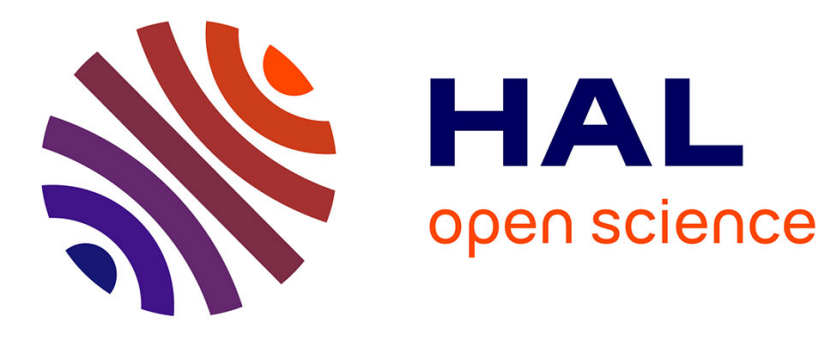

\title{
Fluorescent Self-Assembled Molecular Monolayer on Graphene
}

Sylvain Le Liepvre, Ping Du, David Kreher, Fabrice Mathevet, André-Jean Attias, Céline Fiorini-Debuisschert, Ludovic Douillard, Fabrice Charra

\section{- To cite this version:}

Sylvain Le Liepvre, Ping Du, David Kreher, Fabrice Mathevet, André-Jean Attias, et al.. Fluorescent Self-Assembled Molecular Monolayer on Graphene. ACS photonics, 2016, 3 (12), pp.2291-2296. 10.1021/acsphotonics.6b00793 . hal-01419546

\section{HAL Id: hal-01419546 https://hal.sorbonne-universite.fr/hal-01419546}

Submitted on 19 Dec 2016

HAL is a multi-disciplinary open access archive for the deposit and dissemination of scientific research documents, whether they are published or not. The documents may come from teaching and research institutions in France or abroad, or from public or private research centers.
L'archive ouverte pluridisciplinaire HAL, est destinée au dépôt et à la diffusion de documents scientifiques de niveau recherche, publiés ou non, émanant des établissements d'enseignement et de recherche français ou étrangers, des laboratoires publics ou privés. 
Graphene has focused intensive research in the past ten years due to its unusually high electron mobility, atomic thickness, broadband optical absorption and unique flexibility. ${ }^{1,2,3,4}$ CVD-grown graphene being easily transferable onto arbitrary substrates while preserving high electronic mobilities ${ }^{5}$ it soon appeared as a tantalizing candidate for various applications in photonics ${ }^{6}$, such as solar cells ${ }^{7}$, high-speed photodetectors, ${ }^{8}$ light sources, ${ }^{9}$ ultrafast lasers $^{10}$ or metamaterials ${ }^{11}$. However, as a zero-bandgap semimetal, pristine graphene has a narrow range of roles, merely limited to transparent electrodes. Advanced applications require graphene to be synergistically combined with nanomaterials providing complementary properties.

Recently, non-covalent functionalization of graphene with organic molecular building blocks has appeared as a promising way to modulate its properties in view of functional applications. Actually, graphene provides an atomic-scale crystallographic lattice acting as a template guiding supramolecular self-assembly. ${ }^{12}$ This bottom-up elaboration process, which implies the physisorption onto graphene of mostly planar molecules (tectons), is now well mastered in view of electronic applications. For example, supramolecular self-assembly on CVD grown graphene was used to dope graphene and back-gated graphene field-effect transistor (G-FET) devices were obtained. ${ }^{13}$

Surprisingly, by comparison with electronics, the noncovalent functionalization of graphene is still in its infancy as concerns applications in photonics. Yet, organic dyes offer a high flexibility in the design of innovative photonic devices. Actually, because of their high oscillator strengths, they can play the role of light harvesters, photon sources, exciton funnels, etc. and as such should provide opportunities to enhance or extend the properties of graphene towards light-based applications.

Very recently, the distinctive optical absorption signature of self-assembled dye-graphene architectures, the structure of which were confirmed by scanning tunneling microscopy, have been analyzed by absorption microspectroscopy ${ }^{14}$. However, the main challenge remains the emission of light by such systems, since graphene is known as a strong quencher of electronic excited states through ultrafast non-radiative electron-tunneling exchanges (the so-called Dexter excitation transfer) between the dyes and the graphene ${ }^{15,16,17}$. Hence, the realization of light emitting devices based on dye-graphene assemblies requires to overwhelm Dexter transfers through introduction of an accurately controlled dye-to-graphene electron barrier, for which planar flat-lying molecules are inefficient. Self-assembling building blocks of increasing threedimensional (3D) complexity emerged recently, in particular following the so-called Janus tecton paradigm ${ }^{18,19}$. These systems have been proposed as a platform to attach functional molecular moieties at a controlled distance from graphene ${ }^{20}$.

Here, we demonstrate the first fluorescent supramolecular self-assembly on graphene with a specifically designed 3D Janus tecton. We investigate its twodimensional ordering by scanning tunneling microscopy (STM) and its surface optical properties by absorption and fluorescence spectroscopies. 


\section{Results and discussion}

The $3 \mathrm{D}$ Janus tectons are dual-functionalized and present two faces linked by a rigid spacer. One face is designed so as to steer the self-assembly on graphene and, for the present study, the other is a dye unit ${ }^{18-20}$. More specifically, the dual-functionalized ${ }_{3} \mathrm{D}$ tecton consists of a 3,4,9,10-perylenetetracarboxylic diimide (PTCDI) fluorophore linked to a graphene-binding pedestal by a dithiaparacyclophane pillar and a long leash made of one flexible alkyl chain and a stilbenic unit (scheme 1).

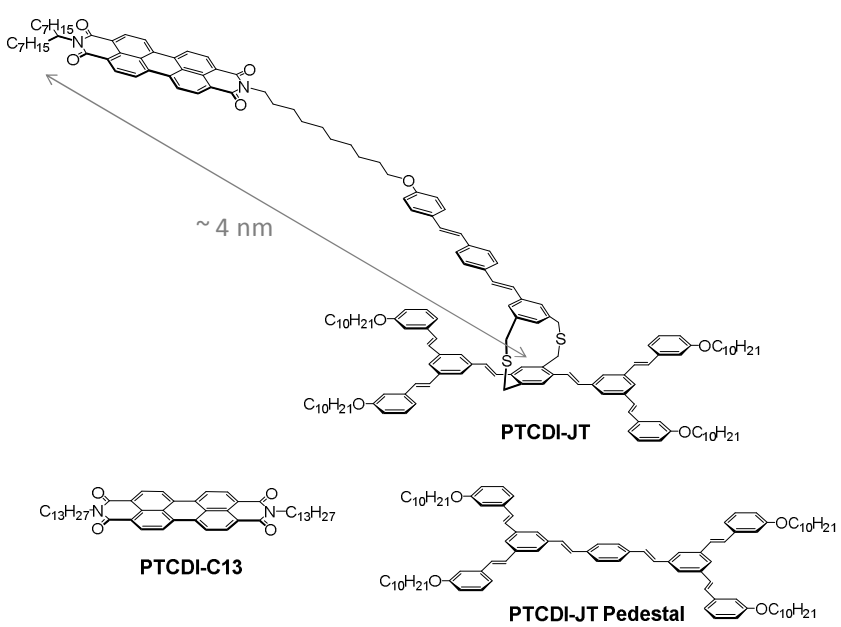

Scheme 1: Schematic structures of the two-faced Janus tecton PTCDI-JT, PTCDI-JT pedestal and N,N'-ditridecyl-3,4,9,10perylenedicarboximide PTCDI- $\mathrm{C}_{13}$ molecules. The distance between the end of the fluorescent unit and the dithiaparacyclophane bridge in PTCDI-JT is typically $\sim 4 \mathrm{~nm}$

The pedestal also prevents the direct adsorption of the dye onto graphene and forms a tunneling barrier for electrons. It is thus expected to slow down Dexter energy transfers from the dye to the graphene layer, a process faster than Förster transfer at such typical $\pi$-stacking distances. ${ }^{21}$
The pedestal unit consists of two molecular clips likely to steer the self-assembly of the whole entity. The clipping motif consists of two $\mathrm{C}_{10} \mathrm{H}_{21}$ alkyl chains which, upon physisorption, lay flat on the surface and aligned along the $<110>$ crystallographic direction of graphene. When two such units interdigitate, their lateral self-assembly leads to the formation of lamellae whose crystallographic structure is equivalent to the one observed upon adsorption of linear alkanes. The resulting enhanced $2 \mathrm{D}$ crystallization energy is at the origin of the binding nature of the $\mathrm{clip}^{15}$. In the following paragraphs, this functional $3 \mathrm{D}$ Janus tecton will be designed as PTCDI-JT.

The spectroscopic properties of PTCDI-JT either in solution or self-assembled on graphene will be compared to those of a model system, namely N,N'-ditridecyl-3,4,9,10perylenedicarboximide (PTCDI-C13). PTCDI-C13 selfassembles on various substrates such as $\mathrm{MoS}_{2}$, graphite (Highly Oriented Pyrolytic Graphite, HOPG) or epitaxial graphene ${ }^{22-24}$.

The self-assembly properties of PTCDI-JT on $\mathrm{sp}^{2}$ hybdridized carbon-based substrates were investigated by STM at the liquid/solid interface at room temperature. The roughness imposed on the CVD graphene monolayer by the underlying polyethylene terephthalate PET substrate makes STM observations difficult due to the poor contrast of molecules compared to this roughness. More accurate observations are obtained with an HOPG substrate and will be preferentially used in what follows. STM data recorded on a graphene substrate, including transferred CVD monolayers, show the equivalence with HOPG and are available in the Electronic Supplementary Information.

The image sequence displayed in figure 1 corresponds to observations recorded at the liquid / HOPG interface for three different solutions: (a) 1:4 ratio solution of PTCDI-JT to the PTCDI-JT pedestal, i.e. the naked precursor clipping unit which constitutes the base of the Janus tecton, (b) an equimolar mixture of the PTCDI-JT and PTCDI-JT pedestal, and (c) a solution of pure PTCDI-JT. a)

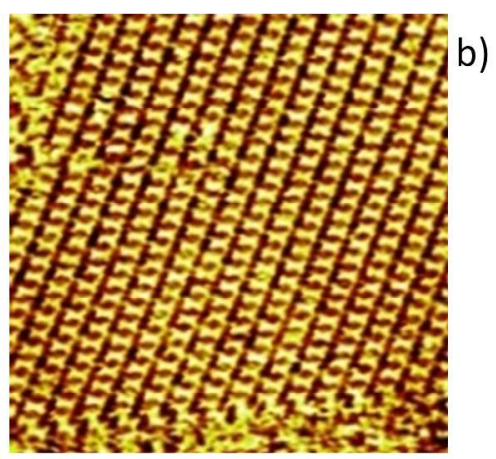

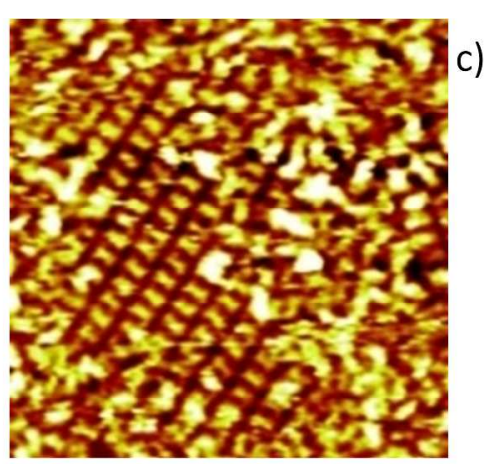

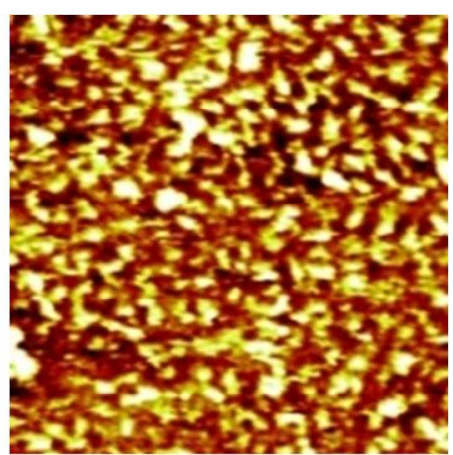

Figure 1. STM images at the liquid/HOPG interface at room temperature, a) 1:4 mixture of Janus tecton and pedestal molecule $(60 \mathrm{~nm} \times 60 \mathrm{~nm}, \mathrm{It}=8.6 \mathrm{pA}, \mathrm{Vt}=-1320 \mathrm{mV}), \mathrm{b}) 1: 1$ mixture of Janus tecton and pedestal molecule $(50 \mathrm{~nm} \times 50 \mathrm{~nm}, \mathrm{It}=9.9 \mathrm{pA}, \mathrm{Vt}$ $=-1602 \mathrm{mV}), \mathrm{c})$ Janus tecton solution $(50 \mathrm{~nm} \times 50 \mathrm{~nm}, \mathrm{It}=8.6 \mathrm{pA}, \mathrm{Vt}=-1596 \mathrm{mV})$. 
The pedestal unit is known to self-organize at the graphite surface forming a well-established twodimensional network with the following parameters: $a=$ $3.8 \mathrm{~nm}, b=2.1 \mathrm{~nm}, \alpha=64^{\circ} .^{25}$ Accordingly, STM images obtained with the 1:4 mixture (figure 1.a) display large domains of a well-ordered arrangement of the clipping molecules of equivalent surface lattice. Experimentally, thanks to a smaller molecule size which reduces its steric constrains, the clipping molecule is shown to selfassemble more easily on graphite than the $3 \mathrm{D}$ Janus tecton.

Upon increase of the PTCDI-JT relative concentration in the applied solution, STM images of the surface display smaller domains of the clipping molecule arrangement together with areas presenting bright spots with rough alignments reminiscent of the underlying ordered regions (figure 1.b). Both domains appear in similar proportions to that in the solution. These misaligned bright features are the signature of the physisorbed PTCDI-JT molecules. Indeed, as far as molecular contrast is concerned, STM favors the signature of aromatic skeletons which present higher electronic surface density of states close to the Fermi level $^{26}$. In the present case, the long and flexible alkyl spacer permits various locations of the PTCDI upper group relative to its pedestal concealing the periodic signature of the clipped pedestals in the horizontal plane. Still, the spatial distribution of these terminal PTCDI groups is not completely random suggesting that neighboring dye groups do aggregate themselves to form fewdye clusters.

For a pure solution of PTCDI-JT, the STM images show a quasi-random distribution of bright spots (figure 1.c) tentatively associated to clusters of aggregated neighboring PTCDI dye moieties and the pedestal network is completely hidden.

To get rough estimates of the dye cluster size and spatial distribution, we built an approximate model based on simple molecular mechanics. Estimating the distance between the end of the fluorescent unit and the dithiaparacyclophane bridge in PTCDI-JT to be $\sim 4 \mathrm{~nm}$ (see scheme 1), we computed the possible number of overlapping fluorescent units in every point of the overlying $3 \mathrm{D}$ space based on the known surface lattice parameters of the two-dimensional clipped network (See figure 2 and figure S2 in Electronic Supplementary Information). In this rough model, the aggregation of independent molecules was modelled with all molecules found at the same location, that is the dimensions of the cluster itself are neglected. This simulation shows the possible aggregation of up to nine fluorescent groups belonging to different neighboring PTCDI-JTs.

Looking at the dimensions of the clipped network surface lattice, the parameter $a$ along the interdigitated alkyl chain direction is approximately twice the parameter $b$ along the lamella axis. Consequently, the dye upper groups are expected to interact more importantly with their respective neighbors along the $b$ direction. One can then reasonably predict the existence of stronger interact- ing subclusters of an even number of molecules. More specifically, the detailed simulation yield a bimodal distribution of the subcluster sizes, whose principal peak corresponds to a cluster size value of $6 \pm 1$. (See Figure $\mathrm{S}_{3}$ in Electronic Supplementary Information).

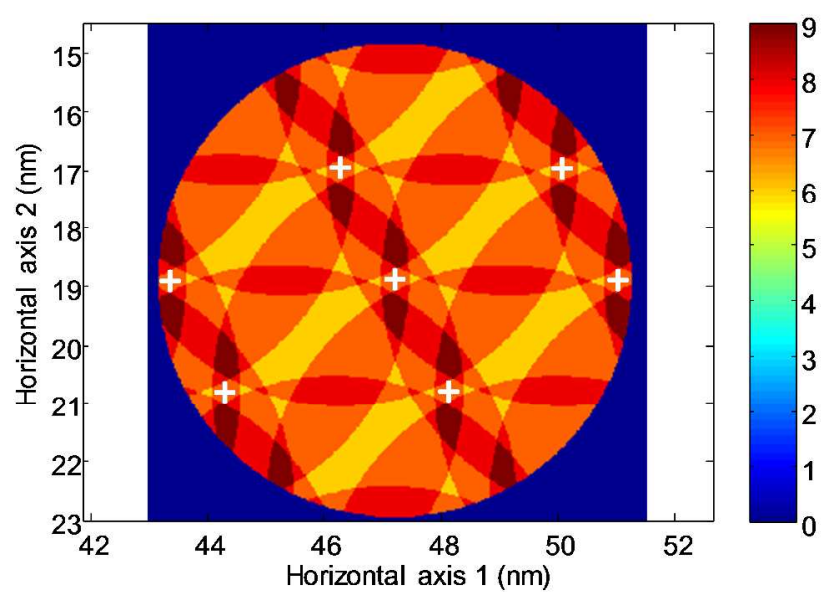

Figure 2. Map of the self-assembled PTCDI-JT tecton indicating the maximum number of aggregated dye-moieties, obtained as the number of intersecting half spheres assuming each dye is located within a 4-nm distance from the center of its pedestal (white crosses).

The most probable location of such 6-dye cluster is computed to be $1.6 \mathrm{~nm}$ above the dithiaparacyclophane rigid pillar and $2.8 \mathrm{~nm}$ or $4.2 \mathrm{~nm}$ away from the bridge in the horizontal plane. The pillar height amounts to about $0.6 \mathrm{~nm}$. The adsorption of the PTCDI-JT pedestal is driven by the physisorption of its aliphatic chains. The aromatic core of the basal unit lies flat on the graphene sheet at a respective distance of $0.33 \mathrm{~nm}^{17}$. So, adopting a static view of the PTCDI-JT arrangement, the clusters of dye moieties lay at about $0.33+0.6+1.6=\mathbf{2 . 5 3} \mathrm{nm}$ above the graphene substrate (See Figure $\mathrm{S}_{4}$ in Electronic Supplementary Information).

Experimental UV-visible absorption spectra of the PTCDI-JT and the PTCDI-C13 molecules in toluene are reported in figure 3 .

The light absorption by the clipping motif manifests itself in the UV range of the spectrum $(\lambda<420 \mathrm{~nm})$ and corresponds to the one recorded for the PTCDI-JT pedestal. The peak at $330 \mathrm{~nm}$ and the shoulder at $375 \mathrm{~nm}$ are assigned respectively to the four terminal stilbene units and to the central distilbene unit. This shoulder in the PTCDIJT absorption spectrum appears to be two times larger in accordance with the presence of another distilbene in the spacer chemical group.

In the visible range $420 \mathrm{~nm}<\lambda<550 \mathrm{~nm}$, the PTCDI-JT absorption matches very well that of the PTCDI- $\mathrm{C}_{13}$ dye. This unaltered spectroscopic signature of the core dye moiety demonstrates that the spacer effectively decouples the conjugated electron systems of fluorophore and pedestal units. 


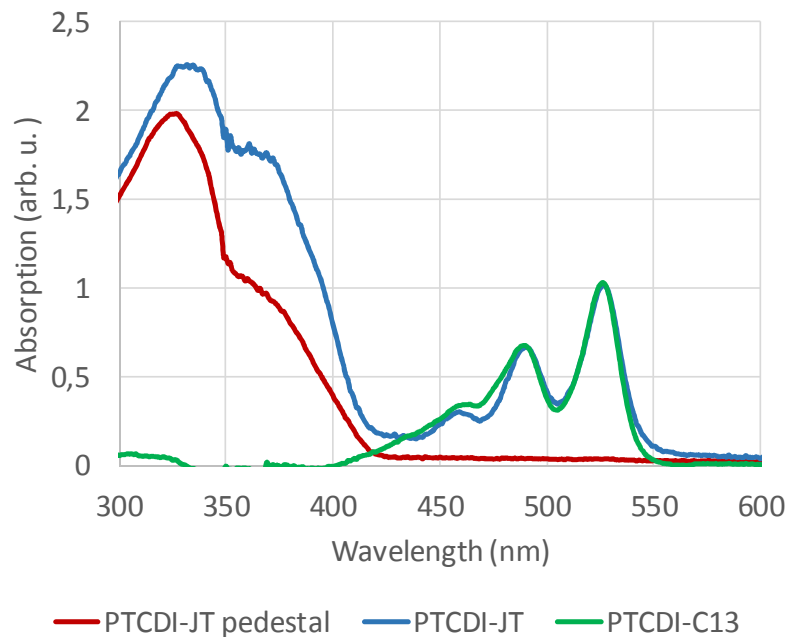

Figure 3. Normalized solution absorption spectra of the PTCDI-JT pedestal, PTCDI-JT, and PTCDI-C13 in toluene.

The of absorption spectra of self-assembled monolayers of PTCDI-JT and PTCDI-C13 on graphite are compared with corresponding toluene solution in figure 4 . The relative peak absorption level of PTCDI-JT monolayer (o.4\%) is nearly 6-times smaller than that of PTCDI-C13 (2.3\%). Considering a similar absorption cross section, a surface density of PTCDI-C13 of $0.5 \mathrm{~mol} / \mathrm{nm}^{2}$ and a $2 / 3$ orientational factor since the PTCDI-C 13 molecules are lying flat on the graphene surface ${ }^{14}$ whereas PTCDI-JT dye moieties are randomly oriented, one can estimate a Janus tecton surface density of $0.12 \pm 0.03$ molecule per $\mathrm{nm}^{2}$. This value is consistent with the surface density extracted from the surface lattice determination by STM of PTCDI-JT self-assembled monolayer on $\mathrm{HOPG}^{19}$ : 0.14 molecules $/ \mathrm{nm}^{2}$. This numerical estimate further supports the conclusion that PTCDI-JT are regularly self-assembled on the graphene surface following the expected binding scheme of the clipping pedestal units.

Both PTCDI-JT and PTCDI-C13 absorption lines drastically change when self-assembled onto graphene as compared with toluene solution (figure 4). Whereas the PTCDI-C 13 absorption merely consists in a rigid red shift compared with the solution, the situation is more complex for PTCDI-JT. Despite a reduced signal-to-noise ratio, an increased weight of higher-order vibronic replicas is perceptible and the rising edge appears less abrupt, which can correspond to a spectral broadening. The redshift observed for PTCDI-C13 is explained by the adsorption of its the aromatic core which lies flat on graphene at a typical $\pi$-stacking distance of $0.33 \mathrm{~nm} .{ }^{14}$ As inferred from STM analysis in the previous section, for PTCDI-JT, the corresponding distance is much larger. According to the static model discussed above, the distance from the dye moieties to the graphene is typically 7 times larger $(\sim 2.5$ $\mathrm{nm}$ ) which prevents such interactions. In contrast with PTCDI-C13, the dye moieties of PTCDI-JT form fewmolecule clusters. The increase of higher-order vibronic peaks is typical of a $\mathrm{H}$-aggregate behavior resulting from a $\pi$-stacking configuration inside clusters ${ }^{27,28}$.

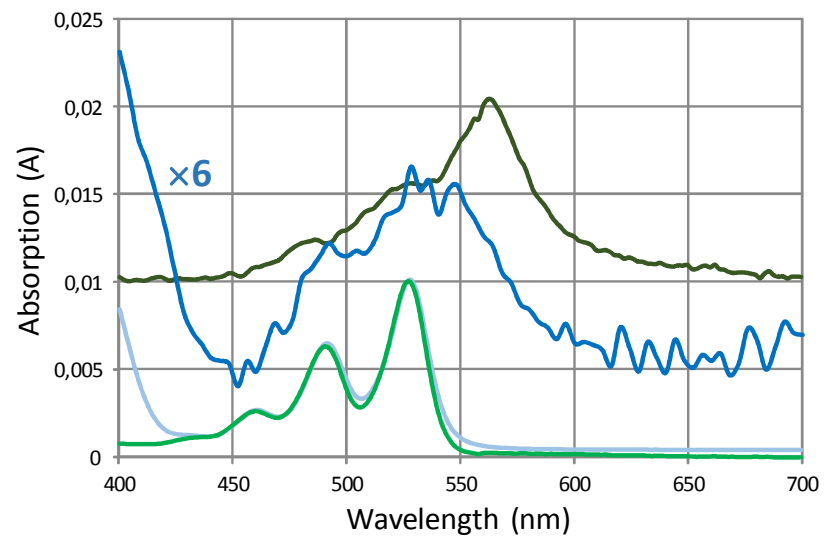

Figure 4. Absorption spectra of PTCDI-JT and PTCDI-C13 self-assembled on graphene. The CVD graphene sheet has been transferred from $\mathrm{Cu}$ onto a transparent PET substrate prior to self-assembly. The measurements were done in transmission, referenced to naked graphene. Because of the absorption of PET in the UV, the spectra are limited to $\lambda>$ $400 \mathrm{~nm}$. The solution spectra of figure 3 are reproduced for an easier comparison.

The spectral broadening of the vibronic spectral features, already present self-assembled PTCDI-C13 is further increased for PTCDI-JT. This probably reflects the inhomogeneity of the dye-moiety assembly as evidenced by STM imaging of the PTCDI-JT tectons which evidences dye clusters with various sizes.

The main result of this work is presented in figure 5 in which the fluorescence signals of the PTCDI-JT molecule self-assembled on graphene is compared with that of the PTCI- $\mathrm{C}_{13}$ reference as recorded in solution, in selfassembled monolayer on graphene or in solid state microcrystals as formed by casting the same PTCDI-C13 toluene solution onto naked fused silica instead of graphene. A photoluminescence signal of the PTCDI-JT selfassembly on graphene is readily measurable whereas no such signal can be detected from the PTCDI-C 13 ordered domains on the same substrate. Hence, the design of PTCDI-JT based on a spacer between the dye and graphene actually reduces the energy transfer rate, as expected. The measured fluorescence quantum yield is still low, estimated in the range of $2 \times 10^{-5}$ to $2 \times 10^{-6}$ by integrating the fluorescence signal between $560 \mathrm{~nm}$ and $745 \mathrm{~nm}$ for an excitation at $532 \mathrm{~nm}$. Since the radiative decay rate of PTCDI is of the order of $10^{-9} \mathrm{~s}^{-1}$, the quenching rate is in the range $10^{14} \mathrm{~s}^{-1}$ to $10^{15} \mathrm{~s}^{-1}$. Although still high, this value is compatible with a residual Förster resonance energy transfer, the electron-exchange quenching process (Dexter transfer) being slower for the distances between dye and graphene that were estimated above. 


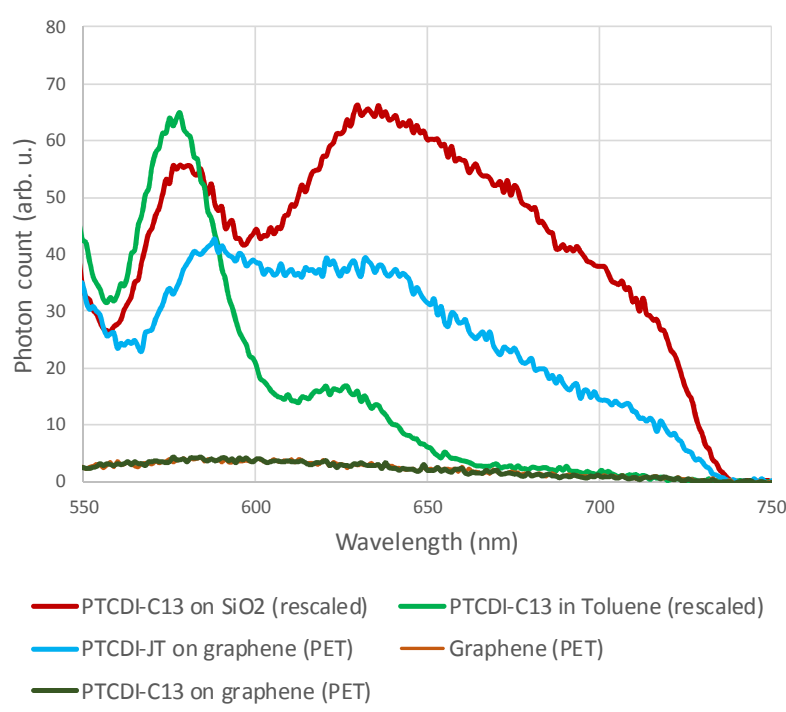

Figure 5. Photoluminescence spectrum of self-assembled PTCDI-JT on graphene (blue) compared with PTCDI-C13 reference signals in toluene (green), self-assembled on graphene (black) and in microcrystal solid state (red). The molecules are excited by a CW laser operating at $532 \mathrm{~nm}$ wavelength which hides the principal fluorescence peak (o - o line) for all the systems.

The formation of clusters also strongly affects the characteristics of luminescence. Although the abovementioned $\mathrm{H}$-aggregate coupling is supposed to slower the radiative decay in favor of the quenching, PTCDI- $\mathrm{C}_{13}$ is fluorescent in the solid state, as shown with the fluorescence of microcrystals formed on silica. Non-radiative relaxations are thus hindered by the strong interactions between stacked molecules. The fluorescence spectrum of PTCDI-JT self-assembled onto graphene (figure 5, blue curve) is intermediate between that of the PTCDI- $\mathrm{C}_{13}$ solvated in toluene (green curve) and that of PTCDI- $\mathrm{C}_{13}$ microcrystals on silica (red curve), showing a progressive displacement of the oscillator strength towards higher vibrational replicas with cluster size, consistently with the $\mathrm{H}$-aggregate-like coupling deduced from absorption spectroscopy. A shorter exciton coherence length in selfassembled clusters is consistent with the involvement of a small number of dye groups, spatially limiting the coherent delocalization of the exciton which is of the order of 2 $\mathrm{nm}$ for similar crystals $^{28,29}$. Furthermore, the $\mathrm{H}$ aggregation geometry could be perturbed from a perfect $\pi$-stacking by the spacer steric hindrance. Instead of being parallel, aromatic cores can adopt an angle that partially decouple the transition dipole moments. This suggests that the controlled formation of few-molecule dye clusters plays a role in the restoration of luminescence. Note that the Janus tecton surface fluorescence peak is slightly red-shifted from the PCTDI-C13 ones consistently with its red-shifted absorption.
In a forthcoming work, lifetime measurement of the radiative decay states will be carried out. This parameter will give insights into the balance between radiative and non-radiative exciton recombination processes.

In summary, we have achieved the first fluorescent molecular self-assembly on graphene. This milestone has been made possible thanks to the development of a $3 \mathrm{D}$ Janus tecton embedding a PTCDI dye uphold away from the substrate plane together with the use of CVD graphene as an optics-friendly $2 \mathrm{D}$ substrate. A fluorescent non-covalent functionalization of graphene could help to alter the surface photonic properties of this zero-gap semiconducting monolayer.

\section{Methods}

Graphene on PET were bought from graphenesupermarket. The self-assembled monolayer has been produced by dipping graphene on quartz in an approximately $10^{-5} \mathrm{~mol} / \mathrm{L}$ Janus tecton solution with toluene as the solvent. To ensure the fabrication of a single monolayer, the sample has been rinsed abundantly with toluene.

The STM images were recorded under ambient conditions (room temperature $\mathrm{T} \sim 300 \mathrm{~K}$ ) with a homemade digital system. Imaging proceeds by the immersion of a $250 \mu \mathrm{m}$ mechanically cut tip of $\mathrm{Pt} / \mathrm{Ir}$ (90/10, Goodfellow) into a $3 \mu \mathrm{L}$ droplet of phenyloctane (Aldrich, 98\%) containing the molecules at a concentration between $10^{-6}$ and $10^{-5} \mathrm{~mol} / \mathrm{L}$ and deposited on a freshly cleaved $10 \mathrm{~mm} \mathrm{x}$ $10 \mathrm{~mm}$ HOPG surface (Goodfellow). The scanning piezoelectric ceramic was calibrated in XY directions with the help of atomically resolved pictures obtained on HOPG. In order to ensure a correct statistical representation of our measurements concerning the structural organization of the monolayers, several images were recorded at different locations of the samples for each molecule of interest. All the images presented were obtained at a quasiconstant current in the variable height mode (the bias voltage VT and the tunneling current IT are indicated in figure captions) and were corrected for the thermal drift by combining two successive images with downward and upward slow-scan directions.

Absorption spectra were recorded on a PerkinElmer Instrument equipped with a tungsten filament white lamp. The sample has been excited with a $532 \mathrm{~nm}$ $\mathrm{CW}$ diode laser through an Olympus objective $(\mathrm{NA}=1.3$, $\times 100)$ and a cooled Andor CCD camera has been used to characterize the fluorescence signals.

Molecular mechanics calculations were performed with a chemical simulation software (Accelrys Discovery Dassault System BioVia).

\section{ASSOCIATED CONTENT}

Supporting Information. Synthesis of PTCDI-JT, STM images on graphene and molecular mechanics calculations. This material is available free of charge via the Internet at http://pubs.acs.org." 


\section{AUTHOR INFORMATION}

\section{Corresponding Authors}

*andre-jean.attias@upmc.fr, fabrice.charra@cea.fr

\section{Author Contributions}

The manuscript was written through contributions of all authors. All authors have given approval to the final version of the manuscript.

\section{Funding Sources}

The authors declare no competing financial interest.

\section{ACKNOWLEDGMENT}

The authors acknowledge the support of the French Agence Nationale de la Recherche (ANR) under grants ANR-13-BS10ooo6 (project SAMPLE) and ANR-14-CE26-0o16 (project SMAL'LED). This work has been supported by a PhD joint funding from CEA and Triangle de la physique.

\section{ABBREVIATIONS}

$2 \mathrm{D}$, two dimensional; $3 \mathrm{D}$, three dimensional; $\mathrm{CCD}$, charge coupled device; CVD, chemical vapor deposition; CW, continuous wave; HOPG, highly ordered pyrolytic graphite; PET, polyethylene terephthalate; PTCDI, 3, 4, 9, 10perylenetetracarboxylic diimide; STM, scanning tunneling microscopy

\section{REFERENCES}

1. Geim, A. K.; Novoselov, K. S., The rise of graphene. $\mathrm{Na}$ ture Materials 2007, 6, 183-191.

2. Geim, A. K., Graphene: Status and Prospects. Science 2009, 324, 1530-1534.

3. Zhang, Y. B.; Tan, Y. W.; Stormer, H. L.; Kim, P., Experimental observation of the quantum Hall effect and Berry's phase in graphene. Nature 2005, 438, 201-204.

4. Novoselov, K. S.; Geim, A. K.; Morozov, S. V.; Jiang, D.; Zhang, Y.; Dubonos, S. V.; Grigorieva, I. V.; Firsov, A. A., Electric field effect in atomically thin carbon films. Science 2004, 306, 666-669.

5. Petrone, N.; Dean, C. R.; Meric, I.; van der Zande, A. M.; Huang, P. Y.; Wang, L.; Muller, D.; Shepard, K. L.; Hone, J., Chemical Vapor Deposition-Derived Graphene with Electrical Performance of Exfoliated Graphene. Nano Letters 2012, 12, 27512756.

6. Bonaccorso, F.; Sun, Z.; Hasan, T.; Ferrari, A. C., Graphene photonics and optoelectronics. Nature Photonics 2010, 4 , 611-622.

7. Wang, X.; Zhi, L.; Mullen, K., Transparent, conductive graphene electrodes for dye-sensitized solar cells. Nano Lett. 2007, 8, 323-327.

8. Mueller, T.; Xia, F.; Avouris, P., Graphene photodetectors for high-speed optical communications. Nature Photon. 2010, 4, 297-301.

9. Matyba, P.; Yamaguchi, H.; Eda, G.; Chhowalla, M.; Edman, L.; Robinson, N. D., Graphene and mobile ions: the key to all-plastic, solution-processed light-emitting devices. ACS Nano 2010, 4, 637-642.

10. Sun, Z. P.; Hasan, T.; Torrisi, F.; Popa, D.; Privitera, G.; Wang, F. Q.; Bonaccorso, F.; Basko, D. M.; Ferrari, A. C., Graphene mode-locked ultrafast laser. ACS Nano 2o10, 4, 803-81o.

11. Wang, B.; Zhang, X.; Garcia-Vidal, F. J.; Yuan, X. C.; Teng, J., Strong Coupling of Surface Plasmon Polaritons in Mon- olayer Graphene Sheet Arrays. Physical Review Letters 2012, 109, o73901.

12. J. M. MacLeod, J. M. ; Rosei, F., Molecular SelfAssembly on Graphene. Small 2013, 10, 1038-1049.

13. Li, B.; Klekachev, A. V.; Cantoro, M.; Huyghebaert, C.; Stesmans, A.; Asselberghs, I.; De Gendt, S.; De Feyter, S.; Toward tunable doping in graphene FETs by molecular self-assembled monolayers. Nanoscale 2013, 5, 9640-9644.

14. Sghaier, T.; Le Liepvre, S.; Fiorini, C.; Douillard, L.; Charra, F., Optical absorption signature of a self-assembled dye monolayer on graphene. Beilstein Journal of Nanotechnology 2016, 7, 862-868.

15. Anger, P.; Bharadwaj, P.; Novotny, L., Enhancement and quenching of single-molecule fluorescence. Physical Review Letters 2006, 96, 113002 .

16. Kasry, A.; Ardakani, A. A.; Tulevski, G. S.; Menges, B.; Copel, M.; Vyklicky, L., Highly Efficient Fluorescence Quenching with Graphene. Journal of Physical Chemistry C 2012, 116, 28582862.

17. Dulkeith, E.; Morteani, A. C.; Niedereichholz, T.; Klar, T. A.; Feldmann, J.; Levi, S. A.; van Veggel, F.; Reinhoudt, D. N.; Moller, M.; Gittins, D. I., Fluorescence quenching of dye molecules near gold nanoparticles: Radiative and nonradiative effects Physical Review Letters 2002, 89, 203002.

18. Bleger, D.; Kreher, D.; Mathevet, F.; Attias, A.-J.; Schull, G.; Huard, A.; Douillard, L.; Fiorini-Debuischert, C.; Charra, F., Surface noncovalent bonding for rational design of hierarchical molecular self-assemblies. Angewandte ChemieInternational Edition 2007, 46, 7404-7407.

19. Du, P.; Jaouen, M.; Bocheux, A.; Bourgogne, C.; Han, Z.; Bouchiat, V.; Kreher, D.; Mathevet, F.; Fiorini-Debuisschert, C.; Charra, F.; Attias, A.-J., Surface-Confined Self-Assembled Janus Tectons: A Versatile Platform towards the Noncovalent Functionalization of Graphene. Angewandte ChemieInternational Edition 2014, 53, 10060-10066.

2o. Bleger, D.; Mathevet, F.; Kreher, D.; Attias, A.-J.; Bocheux, A.; Latil, S.; Douillard, L.; Fiorini-Debuisschert, C.; Charra, F., Janus-Like 3D Tectons: Self-Assembled 2D Arrays of Functional Units at a Defined Distance from the Substrate. Angewandte Chemie-International Edition 2011, 50, 6562-6566.

21. Liu, Q.; Liu, ZF.; Zhong, XY.; Yang, LY.; Zhang, N.; Pan, GL.; Yin, S. G.; Chen, Y.; Wei, J., Polymer Photovoltaic Cells Based on Solution-Processable Graphene and $\mathrm{P}_{3} \mathrm{HT}$. Advanced Functional Materials 2009, 19, 894-904.

22. Yang, H.; Mayne, A. J.; Comtet, G.; Dujardin, G.; Kuk, Y.; Sonnet, P.; Stauffer, L.; Nagarajan, S.; Gourdon, A., STM imaging, spectroscopy and manipulation of a self-assembled PTCDI monolayer on epitaxial graphene. Physical Chemistry Chemical Physics 2013, 15, 4939-4946.

23. Wang, Q. H.; Hersam, M. C., Nanofabrication of Heteromolecular Organic Nanostructures on Epitaxial Graphene via Room Temperature Feedback-Controlled Lithography. Nano Letters 2011, 11, 589-593.

24. Ludwig, C.; Gompf, B.; Petersen, J.; Strohmaier, R.; Eisenmenger, W., STM investigations of PTCDA and PTCDI on graphite and $\mathrm{MoS}_{2}$ - a systematic study of epitaxy and STM image-contrast. Zeitschrift Fur Physik B-Condensed Matter 1994, 93, 365-373.

25. Bleger, D.; Kreher, D.; Mathevet, F.; Attias, A. J.; Arfaoui, I.; Metge, G.; Douillard, L.; Fiorini-Debuisschert, C.; Charra, F., Periodic positioning of multilayered [2.2] paracyclophane-based nanopillars. Angewandte Chemie-International Edition 2008, 47, 8412-8415.

26. Schull, G.; Ness, H.; Douillard, L.; Fiorini-Debuisschert, C.; Charra, F.; Mathevet, F.; Kreher, D.; Attiast, A.-J., Atom substitution for marking and motion tracking of individual mole- 
cules by scanning tunneling microscopy. Journal of Physical Chemistry C 2008, 112, 14058-14063.

27. Ji, H.-F.; Majithia, R.; Yang, X.; Xu, X.; More, K., Selfassembly of perylenedilmide and naphthalenediimide nanostructures on glass substrates through deposition from the gas phase. Journal of the American Chemical Society 2008, 130, 10056-10056.

28. Forrest, S. R., Ultrathin organic films grown by organic molecular beam deposition and related techniques. Chemical Reviews 1997, 97, 1793-1896.
29. Cheng, J.; Wang, W. L.; Mosallaei, H.; Kaxiras, E., Surface Plasmon Engineering in Graphene Functionalized with Organic Molecules: A Multiscale Theoretical Investigation. Nano Letters 2014, 14, 50-56. 
SYNOPSIS TOC (Word Style "SN_Synopsis_TOC"). If you are submitting your paper to a journal that requires a synopsis graphic and/or synopsis paragraph, see the Instructions for Authors on the journal's homepage for a description of what needs to be provided and for the size requirements of the artwork.

Authors are required to submit a graphic entry for the Table of Contents (TOC) that, in conjunction with the manuscript title, should give the reader a representative idea of one of the following: A key structure, reaction, equation, concept, or theorem, etc., that is discussed in the manuscript. Consult the journal's Instructions for Authors for TOC graphic specifications.

\section{Insert Table of Contents artwork here}

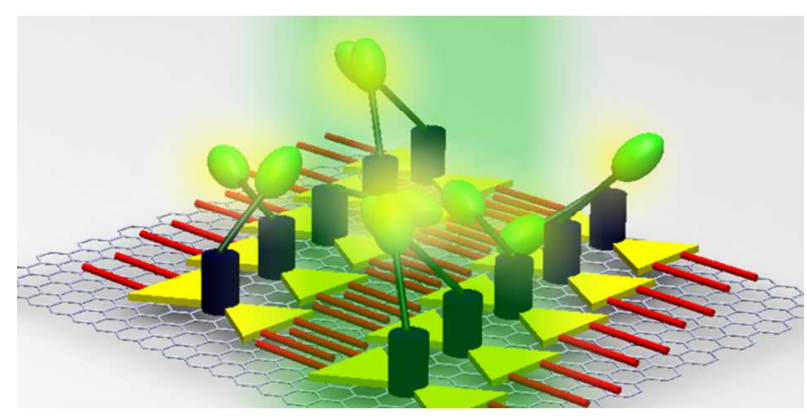

